\title{
Time-Dependent ATR-FTIR Spectroscopic Studies on Solvent Diffusion and Film Swelling in Oil Paint Model Systems
}

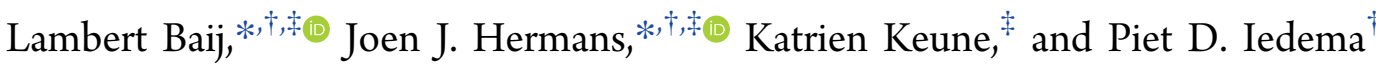 \\ ${ }^{\dagger}$ Van't Hoff Institute for Molecular Sciences, University of Amsterdam, PO Box 94157, 1090GD Amsterdam, The Netherlands \\ ${ }^{\ddagger}$ Conservation and Restoration, Rijksmuseum Amsterdam, PO Box 74888, 1070DN Amsterdam, The Netherlands
}

\section{Supporting Information}

ABSTRACT: In the restoration of paintings, solvent diffusion and swelling of polymeric oil paint binding media are important factors to consider. Common cleaning methods with organic solvents or aqueous solutions could lead to undesirable physicochemical changes in the paint in the long term, though the extent of this effect is not yet clear. We used tailored nonporous model systems for aged oil paint to measure paint swelling and solvent diffusion for a wide range of relevant solvents. Using dynamic mechanical analysis (DMA), the glass transition temperature of

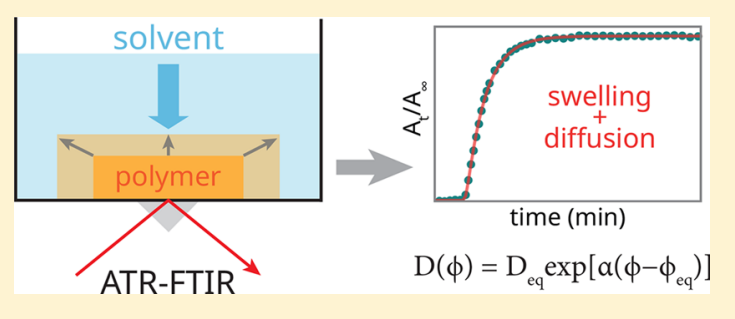
our model systems was found to be close to room temperature. Subsequently, with a custom sample cell and time-dependent attenuated total reflection Fourier transform infrared (ATR-FTIR) spectroscopy, we were able to accurately track swelling and diffusion processes in the polymer films. To quantify the spectroscopic data, we developed a model that completely describes the solvent migration process, including significant film swelling and non-Fickian solvent diffusion. The relation between solvent properties, the diffusion coefficient, and the swelling capacity proved to be rather complex and could not be explained using a single solvent parameter. However, it was found that strongly swelling solvents generally diffuse faster than weakly swelling solvents and that pigmentation does not significantly influence solvent diffusion. These results contribute to a better understanding of transport phenomena in paintings and support the development of improved paint restoration strategies.

\section{INTRODUCTION}

Paintings are exposed to organic solvents or aqueous solutions during restoration or cleaning procedures. These cleaning actions may induce many types of undesirable chemical changes, ${ }^{1-4}$ which are often not immediately visible to the restorer. At present, our understanding of the influence of solvent treatment on fundamental chemical processes is too limited to make a reliable estimate of the long-term effects of common restoration procedures. For example, it is unclear to which depth solvents typically penetrate during cleaning and to what degree soluble components are transported between paint layers. Although the rate of transport processes of soluble components can vary considerably between paintings and cleaning methods, ${ }^{5}$ the degree of paint swelling and the rate of solvent diffusion are both important factors.

The binding medium in oil paint consists of triacylglycerides (TAGs) that have a high degree of unsaturation on their fatty acid chains. As it dries through autoxidation, the oil forms a strongly cross-linked polymer network. ${ }^{6-17}$ The presence of potentially reactive metal-containing pigments and a variety of additives further adds to the complexity of aged oil paint. Oil paints can be considered metastable polymers, subject to slow chemical and physical deterioration processes that affect the appearance and structural integrity of oil paintings.

We have previously demonstrated that mixtures of oil binding media and $\mathrm{ZnO}$ or $\mathrm{PbO}$ gradually form an ionomer-like structure. $^{18,19}$ In such an ionomer structure, metal ions originating from pigments or driers are distributed throughout the polymerized oil network and associated with carboxylic acid groups. An ionomer-like structure has been detected in many oil paint layers that contain lead or zinc ions. We have developed ionomer model systems for aged oil paint to study the molecular structure and dynamic processes in paintings. These models consist of linseed oil copolymerized with lead or zinc sorbate (2,4-hexanedieneoate) complexes, as illustrated in Figure 1. In previous work, small-angle $\mathrm{X}$-ray scattering (SAXS) measurements on these ionomer systems indicated that metal carboxylates form clusters in oil polymers, ${ }^{19}$ and we showed that the reaction of saturated fatty acids with networkbound metal carboxylates is a rapid process that is accelerated by solvent swelling. ${ }^{1}$ In this study of solvent behavior, we have employed the same model systems for aged oil paint (Pbpol and Znpol) and included films of pure polymerized linseed oil ( $\mathrm{pLO}$ ) and films pigmented with $\mathrm{ZnO}$ ( $\mathrm{ZnO}-\mathrm{LO}$ ) for comparison. The pigmented system has the same ionomeric structure in the polymer binding medium as $\mathrm{Znpol}^{19}$ and differs from the pure ionomer system only in the presence of $\mathrm{ZnO}$ pigment particles.

When investigating the solvent diffusion and swelling behavior of a polymer, it is important to know whether the system is studied above or below the glass transition temperature $\left(T_{\mathrm{g}}\right)$. It is well-known that the $T_{\mathrm{g}}$ of polymers

Received: April 26, 2018

Revised: August 17, 2018

Published: September 7, 2018 
Scheme 1. Formation of Ionomers by Copolymerization of Linseed Oil with Metal Sorbate ${ }^{a}$

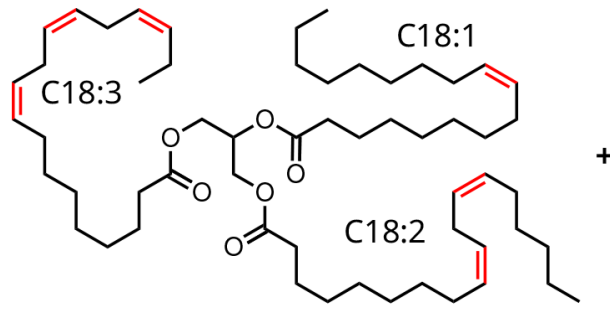

linseed oil TAG unit

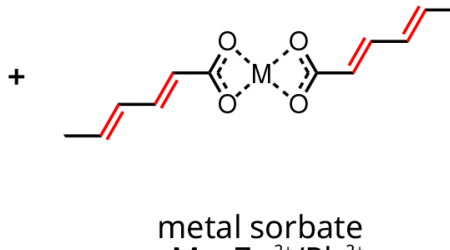

$\mathrm{M}=\mathrm{Zn}^{2+} / \mathrm{Pb}^{2+}$

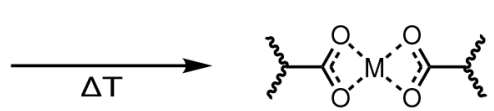

network-bound metal carboxylate

${ }^{a}$ Linseed oil is represented by a typical structure of a triacyl glycerol (TAG) Unit. The depicted carboxylate coordination geometry does not reflect the real (unknown) geometry.

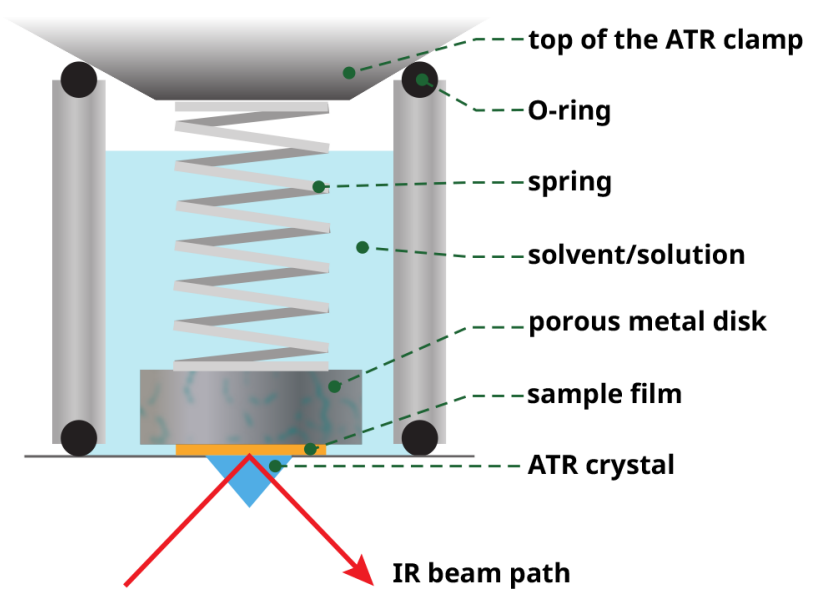

Figure 1. Illustration of the measurement cell used for timedependent ATR-FTIR measurements of polymer films in contact with solvents. The spring provides a small pressure to keep a constant contact between the sample and the ATR crystal as solvents swell the sample, while the porosity of the metal disk ensures unhindered diffusion of the solvent through the sample film.

can strongly influence solvent diffusion behavior. Above the $T_{\mathrm{g}}$ (in the rubbery region), polymers generally show Fickian (ideal) sorption, whereas polymers can show strong deviations from ideal behavior below their $T_{\mathrm{g}}$ (glassy regime). ${ }^{20}$ Therefore, we have studied the viscoelastic properties of our model systems and used dynamic mechanical analysis (DMA) to determine their $T_{\mathrm{g}}$.

Time-dependent attenuated total reflection Fourier transform infrared (ATR-FTIR) spectroscopy was used to follow swelling and diffusion processes in our ionomeric model systems. $^{21}$ This approach has been applied before to study single- or multicomponent solvent diffusion, ${ }^{22-24}$ solute diffusion from an external medium into a polymer, ${ }^{25,26}$ polymer chain interdiffusion, ${ }^{27,28}$ and metal ion diffusion in ionomers. ${ }^{27}$ Diffusion and swelling by organic solvents ${ }^{29-32}$ and water ${ }^{33-37}$ in polymer blends based on linseed oil has been measured before using a variety of methods. For ionomers, data on diffusion and swelling are mainly limited to water and alcohols for fuel cell applications. ${ }^{38-42}$ In most of these studies, polymer films are cast from solution (e.g., by spin-coating or dip-coating) directly onto the ATR elements as thin films to ensure a good contact. However, polymer films based on drying oils are insoluble due to their high degree of crosslinking, and our model systems did not adhere well to ATR elements. To meet the challenge of maintaining a reproducible and constant contact between polymerized oil films and the
ATR crystal during solvent swelling, we developed a measurement setup that can be combined with a standard diamond ATR module in which a very small constant pressure is applied on the sample during solvent exposure. The method allows the measurement of dynamic processes in unsupported film-like materials with at least one smooth surface (e.g., paints, polymers, gels, or skin). Finally, to fully describe the process of solvent sorption in oil-based polymers, we have developed a combined diffusion/swelling model based on a polymer fraction dependent diffusion coefficient.

Combining new insights into the viscoelastic properties of oil paint model systems and the swelling and diffusion behavior of water and other solvents, this work aims to provide a better understanding of solvent action on oil paints to support the development of improved conservation and restoration strategies.

\section{EXPERIMENTAL SECTION}

Sample Preparation. Metal sorbate complexes were synthesized by dissolving $550 \mathrm{mg}$ of sorbic acid (Aldrich, 99+\%) with $1 \mathrm{~mL}$ of triethylamine (Sigma-Aldrich, >99\%) in $20 \mathrm{~mL}$ of demineralized water at $50{ }^{\circ} \mathrm{C}$. The addition of $1.0 \mathrm{~g}$ of $\mathrm{Zn}\left(\mathrm{NO}_{3}\right)_{2} \cdot 6 \mathrm{H}_{2} \mathrm{O}$ (SigmaAldrich p.a.) or $1.1 \mathrm{~g}$ of $\mathrm{Pb}\left(\mathrm{NO}_{3}\right)_{2}$ (Sigma-Aldrich, >99\%) dissolved in $5 \mathrm{~mL}$ of water resulted in immediate precipitation of the white product. After stirring for $20 \mathrm{~min}$, the product was separated by vacuum filtration, washed with water followed by acetone, and dried overnight at reduced pressure. The metal sorbate salts were stored under an inert atmosphere to prevent oxidation.

Binding medium model systems for diffusion studies Znpol-std and Pbpol-std were made by grinding $250 \mathrm{mg}$ of zinc sorbate or an equivalent molar amount of lead sorbate with $1750 \mathrm{mg}$ of coldpressed untreated linseed oil (LO, Kremer Pigmente) to a smooth paste with mortar and pestle. This concentration of metal ions is equivalent to a molar metal carboxylate to ester ratio (COOM/ COOR) of 0.29 and corresponds to roughly $420 \mathrm{mM} \mathrm{Zn}{ }^{2+} / \mathrm{Pb}^{2+}$ in the uncured sample mixture. For DMA analysis, Znpol and Pbpol samples with constant total acid group concentration and increasing metal neutralization were made according to Table S1. The mixtures were applied to $50 \times 75 \mathrm{~mm}^{2}$ glass slides and spread with a drawdown bar to achieve a wet thickness of $190 \mu \mathrm{m}$. The layers were cured overnight in an air-circulated oven at $150{ }^{\circ} \mathrm{C}$, resulting in transparent homogeneous dark orange films with a thickness around $140 \mu \mathrm{m}$. Films of pure polymerized $\mathrm{LO}(\mathrm{pLO})$ were prepared in a similar fashion. Model paint samples for zinc ( $\mathrm{ZnO}-\mathrm{LO})$ were made by grinding $\mathrm{ZnO}$ with cold-pressed untreated $\mathrm{LO}$ in a $1: 1(\mathrm{w} / \mathrm{w})$ ratio to a smooth paste with mortar and pestle. The wet sample thickness was $190 \mu \mathrm{m}$, and the samples were dried at $60^{\circ} \mathrm{C}$ in air for 7 days. For all measurements, $5 \times 5 \mathrm{~mm}^{2}$ squares of the films were cut and lifted off the glass support. The thickness of each sample was measured with a digital micrometer accurate to $1 \mu \mathrm{m}$ prior to diffusion measurements.

Experimental Setup. DMA analysis was performed on a PerkinElmer Pyris Diamond DMA in tensile mode using a 

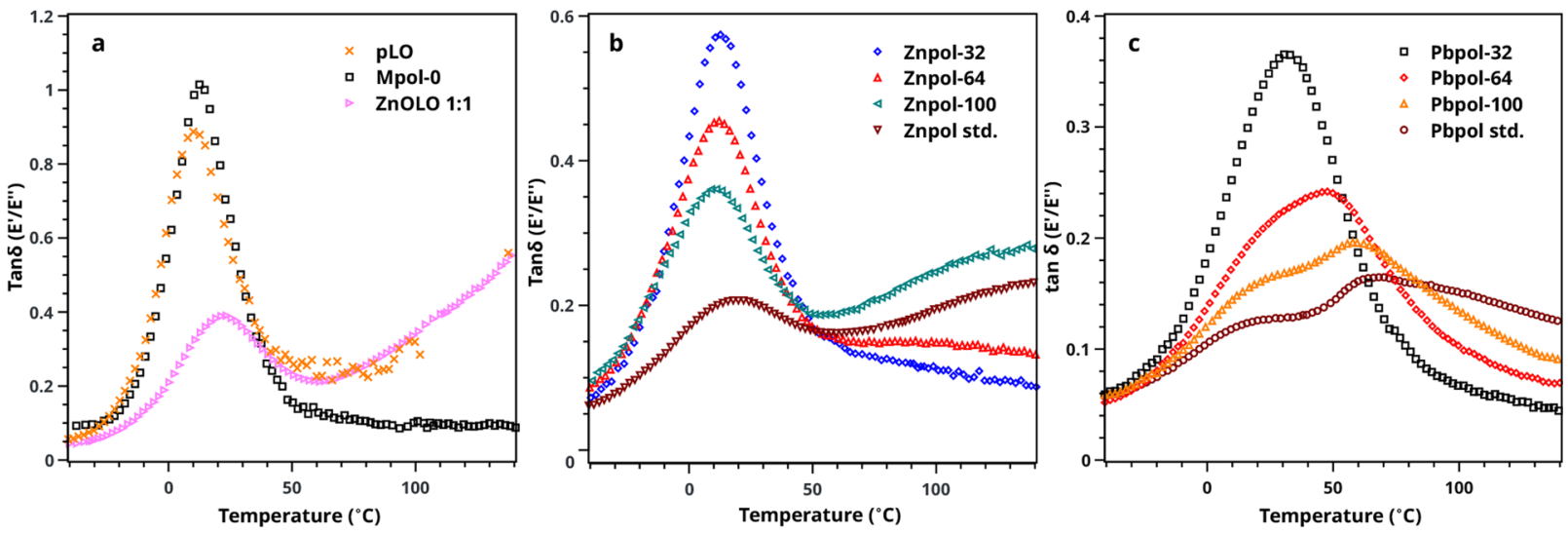

Figure 2. DMA thermograms showing (a) Mpol-0, pLO, and ZnO-LO and (b) Znpol and (c) Pbpol ionomer systems with increasing metal content.

temperature range from -50 to $150{ }^{\circ} \mathrm{C}$ and a heating rate of $2{ }^{\circ} \mathrm{C} /$ min under a $\mathrm{N}_{2}$ atmosphere. For all samples, an amplitude of $10 \mu \mathrm{m}$, a minimum tension/compression force of $10 \mathrm{mN}$, a minimum tension/ compression force gain of $1.1 \mathrm{mN}$, and a force amplitude default value of $500 \mathrm{mN}$ were used. The glass transition temperature $\left(T_{\mathrm{g}}\right)$ was obtained from the maximum value of $\tan \delta$. For Pbpol, two Gaussian functions and a baseline were fitted to determine both $T_{\mathrm{g}}$ and $T_{\mathrm{c}}$ (see Figure S1).

ATR-FTIR spectra were measured on a PerkinElmer Frontier FTIR spectrometer fitted with a Pike GladiATR module with a diamond ATR crystal $(\varnothing=3 \mathrm{~mm})$. Spectra were recorded at the bottom of the sample every 10,30 , or $60 \mathrm{~s}$ (depending on the diffusion rate of the solvent) at $4 \mathrm{~cm}^{-1}$ resolution and averaged over four scans. To measure spectra of polymer samples during exposure to solvents or solutions, a custom-built stainless steel cylinder was used as illustrated in Figure 1. The cell volume was sealed with two solvent-resistant Orings between the top plate and the pressure clamp of the ATR module. The polymer sample was covered by a $\emptyset=10 \mathrm{~mm}$ porous sintered metal disk, and a small but constant pressure was applied to the polymer sample by a spring placed between the pressure clamp of the ATR module and the porous disk. An inlet in the cylinder allowed for the addition of liquids to the sample chamber with a syringe. The inlet was kept sealed with parafilm during measurements to avoid solvent evaporation. In all experiments, analytical grade solvents were used. Automated spectrum collection was started as soon as solvent was injected into the measurement cell.

Data Processing. Diffusion curves in polymer films were measured of cyclohexane $\left(904 \mathrm{~cm}^{-1}\right)$, ethanol $\left(879 \mathrm{~cm}^{-1}\right)$, acetone $\left(529 \mathrm{~cm}^{-1}\right), \mathrm{D}_{2} \mathrm{O}\left(2510 \mathrm{~cm}^{-1}\right)$, methanol- $d_{4}\left(2485 \mathrm{~cm}^{-1}\right), \mathrm{DCM}$ $\left(1265 \mathrm{~cm}^{-1}\right)$, and toluene- $d_{8}\left(541 \mathrm{~cm}^{-1}\right)$. The wavenumbers in parentheses for each solvent refer to the positions of a characteristic solvent band that was sufficiently isolated for accurate band integration. The time-dependent band areas were calculated with PerkinElmer TimeBase software using a baseline anchored to the spectrum to either side of a band and required no further processing before model fitting. FTIR spectra of Znpol at maximum solvent swelling for all solvents are shown in Figure S2. The reproducibility of the diffusion curves was investigated by repeating the acetone diffusion experiment eight times on Znpol films with thicknesses varying between 124 and $159 \mu \mathrm{m}$. The standard deviation in the thickness measurement, caused by uneven sample surfaces, was $\sim 10 \%$. The standard deviation in the swelling factor was determined by repeating the experiment for acetone nine times and was found to be 0.13 . We have applied a method to calculate the $95 \%$ confidence intervals of the fitting parameters using a least-squares procedure ${ }^{43}$ (see the Supporting Information). The $95 \%$ confidence interval in $D_{\text {Fick }}$ resulting from the parameter estimation procedure was below $4 \%$, whereas for $D_{\text {eq }}$ the interval was between $27 \%$ and $34 \%$.
Table 1. $T_{\mathrm{g}}, T_{\mathrm{c}}$, and $\nu_{\mathrm{e}}$ Values for pLO, ZnO-LO, Mpol-0, Znpol, and Pbpol Ionomer Systems with Increasing Metal Content $^{a}$

\begin{tabular}{lcccc}
\multicolumn{1}{c}{ sample } & $\mathrm{COOM} / \mathrm{COOH}$ & $T_{\mathrm{g}}\left({ }^{\circ} \mathrm{C}\right)$ & $T_{\mathrm{c}}\left({ }^{\circ} \mathrm{C}\right)$ & $\nu_{e}\left(\mathrm{~mol} / \mathrm{cm}^{3}\right)$ \\
pLO & 0 & 8 & & $2.1 \times 10^{-5}$ \\
Mpol-0 & 0 & 10 & & $3.5 \times 10^{-4}$ \\
Pbpol-32 & 0.32 & 33 & 31 & $8.5 \times 10^{-4}$ \\
Pbpol-64 & 0.64 & 20 & 53 & $1.2 \times 10^{-3}$ \\
Pbpol-100 & 1 & 17 & 61 & $4.0 \times 10^{-3}$ \\
Pbpol-std & 1 & 11 & 67 & $4.8 \times 10^{-3}$ \\
Znpol-32 & 0.32 & 11 & & $6.3 \times 10^{-4}$ \\
Znpol-64 & 0.64 & 10 & & $6.8 \times 10^{-4}$ \\
Znpol-100 & 1 & 9 & & $8.6 \times 10^{-4}$ \\
Znpol-std & 1 & 16 & & $2.2 \times 10^{-3}$ \\
ZnO-LO & & 21 & & $5.6 \times 10^{-4}$
\end{tabular}

${ }^{a} \mathrm{COOM} / \mathrm{COOH}$ refers to the proportion of sorbic acid groups that is coordinated to a metal ion $\left(\mathrm{Zn}^{2+}\right.$ or $\left.\mathrm{Pb}^{2+}\right)$.

\section{RESULTS AND DISCUSSION}

DMA Analysis of Model Paint Films. The viscoelastic properties of the model ionomer and paint systems were characterized by DMA. Figure 2 and Table 1 present glass transition temperature and cross-link density data for ionomer films with increasing metal content (from $0 \%$ neutralization to $100 \%)$ as well as pure polymerized linseed oil (pLO) and $\mathrm{ZnO}$-based paint ( $\mathrm{ZnO}-\mathrm{LO})$. The $T_{g}$ values were obtained from the maximum of the $\tan \delta$ plots (note that $\tan \delta=E^{\prime \prime} / E^{\prime}$, where $E^{\prime \prime}$ and $E^{\prime}$ are the loss and storage modulus values, respectively).

At present, there is some uncertainty in the literature on the $T_{\mathrm{g}}$ of linseed oil-based paint films. Previous studies reported values between 0 and $10{ }^{\circ} \mathrm{C},{ }^{44-47}$ while Ploeger et al. $^{48}$ observed an increase to around $40{ }^{\circ} \mathrm{C}$ upon aging of the paint films. LO polymers modified with styrene ${ }^{49}$ or epoxy groups ${ }^{50}$ tend to have a higher $T_{\mathrm{g}}$. We found a $T_{\mathrm{g}}$ for $\mathrm{pLO}$ at $8{ }^{\circ} \mathrm{C}$. The $T_{\mathrm{g}}$ is raised slightly to $10{ }^{\circ} \mathrm{C}$ upon copolymerization with sorbic acid (Mpol-0, essentially an "empty" ionomer), suggesting possible hydrogen bonding between adjacent acid groups. $^{51}$

For the lead ionomers ( $\mathrm{Pbpol})$, the characteristic behavior of a two-phase system was observed. It is known that in a relatively apolar polymer matrix the introduction of ionic groups can lead to the formation of ionic aggregates, ${ }^{52,53}$ giving rise to ion-poor (i.e., polymer matrix) and ion-rich (i.e., metal 
carboxylate cluster region) phases. The $\tan \delta$ peak at low temperature is attributed to the bulk $T_{g}$, while the peak at higher temperatures corresponds to the glass transition in the ion-rich regions $\left(T_{c}\right)$. This $T_{c}$ peak grows at the expense of the main glass transition peak and shifts to higher temperatures as the ion content increases. In contrast to $T_{\mathcal{c}}$, the "matrix" $T_{\mathrm{g}}$ for Pbpol shifts to lower temperatures when the ion content is increased. The shifts in $T_{\mathrm{g}}$ and $T_{\mathrm{c}}$ suggest that there are changes in the structure or composition of the ion-poor and ion-rich phases in Pbpol with increasing lead content, not just in the concentration of ion-rich clusters. In previous research, SAXS analysis on Pbpol samples showed that long-range inhomogeneity in lead concentration exists at low neutralization levels, while lead ions tended to be grouped in smaller and more defined ion-rich clusters at high neutralization. ${ }^{19}$ In line with these observations, to explain the trends in $T_{\mathrm{g}}$ and $T_{\mathrm{c}}$ we propose that the lead carboxylate concentration (and therefore also the cross-link density) decreases in the bulk polymer with increasing neutralization leading to lower $T_{\mathrm{g}}$ values, while at the same time the ion-rich phases become more enriched in lead, leading to a rise in $T_{\mathrm{c}}$.

Considering Znpol, it was observed that increasing the zinc content has no significant effect on the $T_{\mathrm{g}}$ values. No $T_{\mathrm{c}}$ was observed for Znpol in the measured temperature range, though Figure $2 \mathrm{~b}$ seems to suggest there could be a transition $(>150$ $\left.{ }^{\circ} \mathrm{C}\right)$ that is related to ion cluster relaxation. A very high $T_{c}$ would be in agreement with the fact that the formation of crystalline zinc soaps in ionomeric polymer systems is much slower than the formation of lead soaps. ${ }^{1,54}$ The $\tan \delta$ peaks close to room temperature decreased strongly in height with increasing zinc content, which is thought to reflect a decreasing mobility of the polymer chain segments. ${ }^{55}$ While decreasing chain mobility seems plausible with increasing zinc carboxylate content, SAXS measurements on Znpol systems showed no clear trend in either the concentration or the size of ionic domains with increasing zinc neutralization. ${ }^{19}$ Clearly, more DMA and SAXS experiments on systems with a wide neutralization range are necessary to clarify the structural details of linseed oil-based ionomers.

The $T_{\mathrm{g}}$ of $\mathrm{ZnO}-\mathrm{LO}$ was measured at $21{ }^{\circ} \mathrm{C}$, even though Phenix et al. ${ }^{46}$ could only detect "progressive, general thermal softening" in 16 year old $\mathrm{ZnO}$ paint. The slightly higher $T_{\mathrm{g}}$ for $\mathrm{ZnO}$-LO might be explained by the lower degree of curing for $\mathrm{ZnO}-\mathrm{LO}\left(1\right.$ week at $60{ }^{\circ} \mathrm{C}$ ) compared to $\mathrm{Znpol}(17 \mathrm{~h}$ at 150 $\left.{ }^{\circ} \mathrm{C}\right)$.

The elasticity modulus $E^{\prime}$ in rubbery plateau region at 140 ${ }^{\circ} \mathrm{C}$ (see Figure S3) was used to calculate cross-link densities $\left(\nu_{\mathrm{e}}\right)$ according to ${ }^{56}$

$$
\nu_{\mathrm{e}}=\frac{E^{\prime}}{3 R T}
$$

The calculated values for $\nu_{\mathrm{e}}$ as a function of metal carboxylate concentration (COOM/COOR) are shown in Figure 3. For both Znpol and Pbpol, $\nu_{\mathrm{e}}$ increases significantly with metal content. Ionic cross-links thus contribute significantly to the measured storage modulus and calculated cross-link densities, despite the fact that the unbound carboxylic acids in the not fully neutralized ionomers can also form "cross-links" by hydrogen bonding. In analogy with Weiss et al., ${ }^{57}$ we also calculated a cross-link density by assuming that every metal sorbate unit forms an additional cross-link $\left(\nu_{c}\right)$ on top of the cross-link density of Mpol-0 and compared this with $\nu_{\mathrm{e}}$ calculated from eq 1 . It was found that $\nu_{\mathrm{e}}$ was consistently

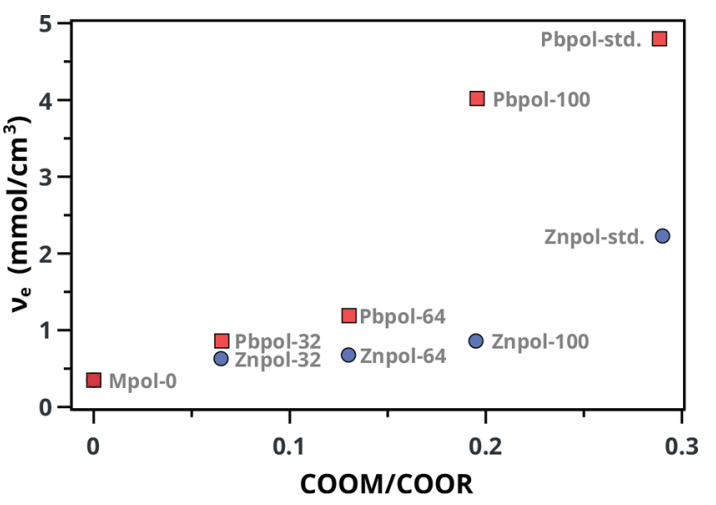

Figure 3. Relation between ion content and $\nu_{\mathrm{e}}$ as calculated by eq 1 .

higher than $\nu_{\mathrm{c}}$ by approximately $5-20 \%$. In the paper by Weiss et al., ${ }^{57}$ two possible explanations are given for this high contribution of metal neutralization to $\nu_{\mathrm{e}}$ : (1) Synergism exists between the ionic cross-links and molecular entanglements that yield a higher effective cross-link density than that based on a linear combination of the two effects. (2) Ionic clusters have a lower molar mass between cross-links than that calculated from the average chain length between simple contact ion-pair associations. Because we are currently primarily concerned with solvent behavior in these oil-based ionomers, the in-depth investigation of these hypotheses is beyond the scope of this paper.

Concluding, we established that our model systems have bulk $T_{\mathrm{g}}$ values close to room temperature. Therefore, it is expected that both Znpol and Pbpol will show Fickian-type solvent diffusion behavior. The effect of the $T_{c}$ on solvent diffusion remains to be investigated.

Solvent Diffusion and Swelling in Model Paint Films. The diffusion and swelling behavior of seven solvents was investigated in Znpol, Pbpol, and ZnO-LO model systems. Solvent concentration profiles were obtained by integration of characteristic solvent IR bands. The maximum swelling capacity of each solvent was determined by monitoring the IR absorption bands corresponding to the linseed oil polymer. As solvent diffused into the volume probed by the IR beam, the concentration of polymer chains in that volume decreased according to a profile that mirrored the solvent diffusion profiles. Because it was shown previously ${ }^{58}$ that FTIR spectroscopy can be used to measure swelling factors that are equivalent to those obtained from size measurements using a CCD camera, the relative difference in absorbance of the ester carbonyl band $\left(1740 \mathrm{~cm}^{-1}\right)$ at saturation and at $t=($ i.e., $\left.\Delta A / A_{0}\right)$ was used as a measure for the maximum degree of swelling $\left(f_{\text {eq }}\right)$. Values for $f_{\text {eq }}$ for all solvents and samples are given in Table 2.

A significant variation in the solvent swelling capacity was observed. DCM swelled the investigated model systems by more than a factor of 2 , while water hardly swelled the oil polymer at all (approximately 5-10\% volume increase for zinc and lead). The FTIR spectroscopic method for measuring equilibrium swelling accurately reproduced trends reported in the literature for solvent swelling of oil paints and confirms the low swelling capacity of water. ${ }^{59,60}$ The trends in $f_{\text {eq }}$ for different solvents are consistent between all three investigated polymers. It is noted that for low-swelling solvents such as cyclohexane and water the differences between Znpol and $\mathrm{Pbpol}$ are larger. In the case of cyclohexane in Pbpol, it was not 
Table 2. Values of Diffusion Coefficient at Equilibrium Swelling According to the Diffusion-Swelling Model ( $\left.D_{\text {eq }}\right)$ and Those with the Assumption of Fickian Diffusion $\left(D_{\text {Fick }}\right)^{a}$

\begin{tabular}{|c|c|c|c|c|c|c|c|c|c|}
\hline \multirow[b]{2}{*}{ solvent } & \multicolumn{3}{|c|}{ Znpol } & \multicolumn{3}{|c|}{ Pbpol } & \multicolumn{3}{|c|}{ ZnO-LO } \\
\hline & $D_{\text {eq }}\left(10^{-11} \mathrm{~m}^{2} / \mathrm{s}\right)$ & $D_{\text {Fick }}\left(10^{-11} \mathrm{~m}^{2} / \mathrm{s}\right)$ & $f_{\text {eq }}$ & $D_{\text {eq }}\left(10^{-11} \mathrm{~m}^{2} / \mathrm{s}\right)$ & $D_{\text {Fick }}\left(10^{-11} \mathrm{~m}^{2} / \mathrm{s}\right)$ & $\overline{f_{\text {eq }}}$ & $D_{\text {eq }}\left(10^{-11} \mathrm{~m}^{2} / \mathrm{s}\right)$ & $D_{\text {Fick }}\left(10^{-11} \mathrm{~m}^{2} / \mathrm{s}\right)$ & $f_{\text {eq }}$ \\
\hline DCM & 57 & 14 & 1.2 & 48 & 14 & 1.1 & 46 & 16 & 0.66 \\
\hline acetone & 15 & 4.9 & 0.73 & 27 & 6.1 & 0.89 & 16 & 7.5 & 0.47 \\
\hline toluene- $d_{8}$ & 7.9 & 2.5 & 0.71 & 7.9 & 3.1 & 0.81 & 3.8 & 2.2 & 0.26 \\
\hline methanol- $d_{4}$ & 3.7 & 1.0 & 0.60 & 1.8 & 0.72 & 0.56 & 2.3 & 1.5 & 0.24 \\
\hline ethanol & 1.6 & 0.43 & 0.89 & 1.2 & 0.40 & 0.87 & 1.1 & 0.50 & 0.41 \\
\hline cyclohexane & 0.28 & 0.14 & 0.24 & $0.26^{b}$ & $0.11^{b}$ & $0.45^{b}$ & 0.086 & 0.052 & 0.18 \\
\hline $\mathrm{D}_{2} \mathrm{O}$ & 0.059 & 0.061 & 0.05 & 0.064 & 0.053 & 0.13 & 0.038 & 0.035 & 0.05 \\
\hline
\end{tabular}

${ }^{a}$ Diffusion parameters have been estimated at a $95 \%$ confidence interval and were found to vary between $27 \%$ and $34 \%$ for $D_{\text {eq }}$ and below $4 \%$ for $D_{\text {Fick }}$ (see the Supporting Information). The standard deviation in the swelling factor is 0.13 . ${ }^{b}$ Measured at $40{ }^{\circ} \mathrm{C}$.

possible to carry out the diffusion experiment at room temperature due to detachment of the rather stiff polymer film from the ATR crystal during solvent exposure. Therefore, this measurement was conducted at $40{ }^{\circ} \mathrm{C}$. Despite the differences in viscoelastic properties that were measured with DMA, no significant differences in $f_{\text {eq }}$ between Znpol and Pbpol were found (standard deviation in $f_{\text {eq }}$ of \pm 0.13 , see Experimental Section). However, $\mathrm{ZnO}-\mathrm{LO}$ did exhibit consistently lower values for $f_{\text {eq }}$ than the unpigmented ionomer films. This result is explained by the fact that the pigment particles do not contribute to the swelling of the system upon solvent absorption.

Fickian Diffusion Model. A Fickian diffusion model for ATR-FTIR has been developed by Fieldson and Barbari, ${ }^{21}$ which allows for the calculation of diffusion parameters using the recorded IR spectra. Having established that the model systems under investigation have $T_{\mathrm{g}}$ values close to room temperature, we investigated the applicability of this Fickian diffusion model. A typical concentration profile of acetone in

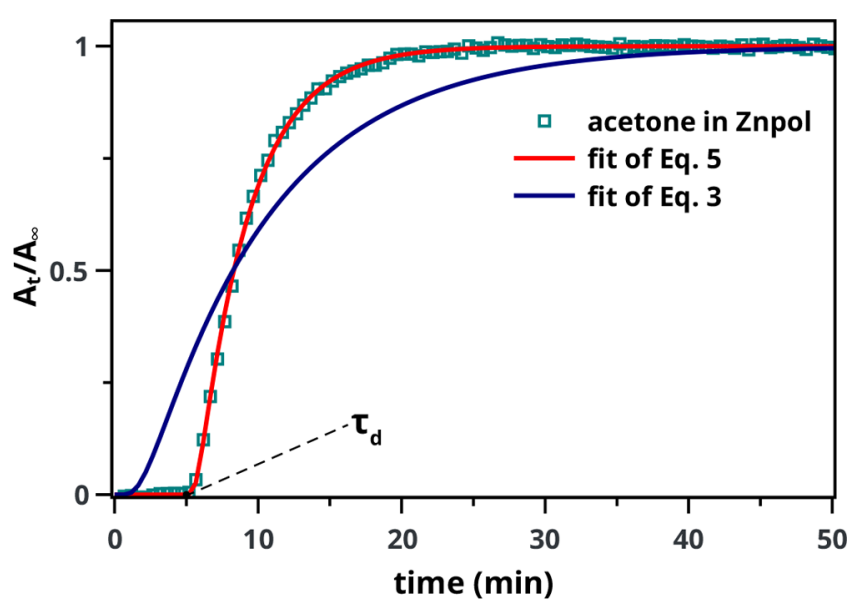

Figure 4. Diffusion profile for acetone in Znpol (square symbols) and fits of a standard (blue) and adapted (red) Fickian diffusion model to the data.

Znpol is shown in Figure 4. For ideal diffusion with a constant diffusion coefficient, Fick's second law states

$$
\frac{\partial C}{\partial t}=D\left(\frac{\partial^{2} C}{\partial x^{2}}\right)
$$

where $C$ is the concentration of penetrant in the medium and $D$ is the diffusion coefficient. A solution to this differential equation has been derived by Fieldson and Barbari ${ }^{21}$ using appropriate boundary conditions for the geometry of an ATRFTIR setup, making use of the exponential decay of the evanescent field of the IR beam into the sample and the BeerLambert law

$$
\begin{aligned}
& \frac{A(t)}{A(\infty)}=1-\frac{8 \gamma}{\pi(1-\exp [-2 \gamma \delta])} \times \\
& \sum_{n=0}^{\infty}\left[\frac{\exp \left[-D f^{2} t\right]\left(f \exp [-2 \gamma \delta]+2 \gamma(-1)^{n}\right)}{(2 n+1)\left(4 \gamma^{2}+f^{2}\right)}\right]
\end{aligned}
$$

in which

$$
f=\frac{(2 n+1) \pi}{2 \delta}
$$

and

$$
\gamma=\frac{2 n_{2} \pi}{\lambda} \sqrt{\sin ^{2} \theta-\left(\frac{n_{1}}{n_{2}}\right)^{2}}
$$

In this relation, $\gamma$ is the inverse of the penetration depth $d_{\mathrm{p}}$ of the IR beam into the sample. $d_{\mathrm{p}}$ varies from 0.5 to $3.5 \mu \mathrm{m}$ from 3500 to $500 \mathrm{~cm}^{-1}$ in our experiments, so with a typical film thickness around $150 \mu \mathrm{m}$ the recorded spectra are representative for the bottom side of the film. Parameters $n_{1}$ and $n_{2}$ are the refractive indices of the polymer sample and ATR crystal, respectively, $\delta$ is the thickness of the film, $\theta$ is the angle of incidence of the IR beam $\left(45^{\circ}\right)$, and $\lambda$ is the wavelength corresponding to the maximum of the characteristic IR band of the penetrant. For the experimental conditions described in this paper, the shape of the diffusion profile is very insensitive to the exact value of $\gamma$. Therefore, a single measured value for the refractive index of a Znpol film was assumed as a constant for all calculations. This model represents an ideal case of penetrant transport (i.e., negligible preferential interaction between the polymer and solvent), corresponding to a diffusion of penetrant that is on a much slower time scale than polymer chain relaxation. ${ }^{20}$

Fitting eq 3 to the diffusion curve of acetone in Znpol, it is immediately clear that the ideal model is not capable of describing simultaneously the long delay until solvent signal is first detected as well as the sharp increase in solvent concentration that follows (see Figure 4, blue curve). Similar results were found for all investigated solvents and films (for instance, see Figure S4). While eq 3 is frequently applied to 


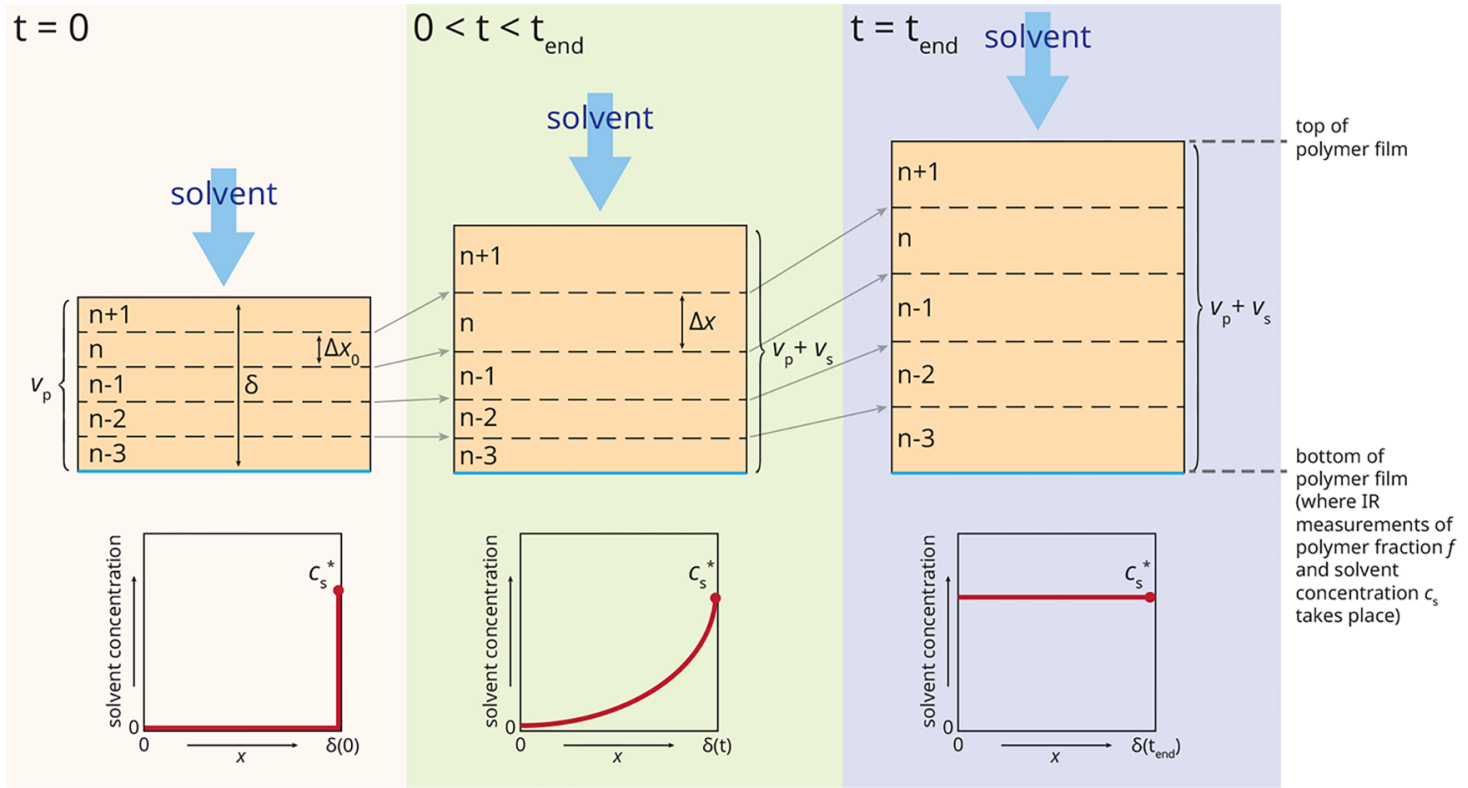

Figure 5. Illustration of the method-of-lines model with elements that increase in thickness during swelling. The concentration $c_{\mathrm{s}}^{*}$ is the concentration of solvent at equilibrium or maximum swelling.

describe penetrant diffusion in polymers, our use of relatively thick films enhances the effect of potential polymer swelling during diffusion, and it causes a significant delay time $\left(\tau_{\mathrm{d}}\right.$, the time necessary for solvent to reach the sampling volume). Therefore, using thick polymer films, it becomes easier to investigate deviations from ideal diffusion behavior.

To allow for the considerable delay time, $\tau_{\mathrm{d}}$, eq 3 was adapted such that

$$
\frac{A(t)}{A(\infty)}= \begin{cases}0, & \text { for } 0<t<\tau_{\mathrm{d}} \\ \frac{A\left(t-\tau_{\mathrm{d}}\right)}{A(\infty)}, & \text { for } t \geq \tau_{\mathrm{d}}\end{cases}
$$

With this procedure, the swelling between $0<t<\tau_{\mathrm{d}}$ is neglected, while Fickian diffusion is assumed at $t>\tau_{\mathrm{d}}$. The shifted Fickian model of eq 5 provided a near-perfect fit to the experimental concentration profiles, as shown in Figure 4. Corresponding diffusion coefficients $D_{\text {Fick }}$ are listed in Table 2.

From the excellent match between the ideal diffusion model and the experimental concentration profiles at $t>\tau_{\mathrm{d}}$, it can be concluded that once the polymer is nearly completely swollen (i.e., only minor changes in polymer fraction), (near) Fickian diffusion behavior is observed in the measurement volume at the bottom of the film. However, film swelling does cause an significant increase in $\tau_{\mathrm{d}}$. This effect is easily observed by comparing the experimental $\tau_{\mathrm{d}}$ values with values calculated with the common relation between $\delta$ and $D, \delta=\sqrt{D \tau_{\mathrm{d}}}$. The relation yields consistently lower $\tau_{\mathrm{d}}$ values than observed experimentally, demonstrating that the ideal diffusion model needs to be replaced with a diffusion model that accounts for film swelling.

In paint restoration, the swelling of paintings is an important phenomenon. It is well-known that aged oil paint can swell significantly during cleaning and varnish removal. ${ }^{61}$ Especially with the relatively short solvent application times typical for restoration work, one might argue that equilibrium swelling $\left(f_{\text {eq }}\right)$ is never reached, except in the areas of the paint closest to the surface. The initial stages of solvent diffusion are therefore especially relevant to understand the potential effects of restoration treatments on paintings, adding to the importance of developing a model that describes the entire swelling and diffusion process.

Diffusion-Swelling Model. Construction of the Diffusion-Swelling Model. In the model paint systems, sample swelling to more than twice the original dry volume occurred in the most extreme cases, corresponding to a polymer fraction decreasing from 1 to 0.5 . Solvent swelling and diffusion in polymers is widely discussed in the literature. ${ }^{62-66}$ The most comprehensive models are those based on the free-volume theory originally developed by Vrentas and Duda. ${ }^{67,68}$ However, free-volume models contain many parameters that despite having physical meaning, are largely unknown. Therefore, we decided to employ the simplest model that accounts for a decreasing polymer volume fraction during swelling, using a variable diffusion coefficient. The functional form of this model is inspired on Cukier's formula for diffusion in hydrogels ${ }^{64}$ and describes a diffusion coefficient that is an exponential function of the polymer volume fraction $\phi$ as

$$
D=D_{0} \mathrm{e}^{\alpha \phi^{\beta}}
$$

Because $D=D_{0}$ at $\phi=0$ (pure solvent), for hydrogels $D_{0}$ has been interpreted as the diffusion coefficient of solvent molecules in their own environment ${ }^{65}$ or at infinite dilution. ${ }^{64}$ However, for systems swelling much less than hydrogels, the pre-exponential factor $D_{0}$ should be considered as a fitting parameter. Parameter $\alpha$ has been correlated to the radius of solvent molecules. ${ }^{64}$ For parameter $\beta$, values of 0.5 and 0.75 are mentioned by Amsden. ${ }^{64}$ Given the limited range in polymer fraction $\phi$ for our samples, it was expected that one parameter describing its effect of diffusion would be sufficient, and we assumed $\beta=1$, yielding a diffusion coefficient dependent only on $D_{0}$ and $\alpha$. As the diffusion coefficient at equilibrium swelling, $D_{\text {eq }}$, is most relevant in the present case, eq 6 is rewritten as

$$
D=D_{\text {eq }} \mathrm{e}^{\alpha\left(\phi-\phi_{\text {eq }}\right)}
$$


Here, $\phi_{\text {eq }}$ is the polymer fraction at equilibrium (complete) swelling $f_{\text {eq }}$. Next, the swelling factor $F$ is defined as

$$
F=1 / \phi=v_{\text {tot }} / v_{\mathrm{p}}=\left(v_{\mathrm{s}}+v_{\mathrm{p}}\right) / v_{\mathrm{p}}
$$

Here, $v_{\mathrm{s}}$ is volume of solvent, $v_{\mathrm{p}}$ is volume of polymer, and $v_{\text {tot }}$ is the total volume. The total polymer mass is assumed to occupy the same (partial) volume, $v_{\mathrm{p}}$, during swelling, while $v_{\mathrm{s}}$ and $v_{\text {tot }}$ do increase. If $c_{\mathrm{s}}$ is the molar solvent concentration, then $v_{\text {tot }}$ contains $v_{\text {tot }} c_{\mathrm{s}}$ moles of solvent of volume $v_{s}$. Given the solvent density $\rho_{\mathrm{s}}$ and the solvent molar mass $M_{\mathrm{s}}$, we know that $v_{\mathrm{s}}$ contains $v_{\mathrm{s}} \rho_{\mathrm{s}} / M_{\mathrm{s}}$ moles of of solvent. Therefore

$$
v_{\text {tot }} c_{\mathrm{s}}=v_{\mathrm{s}} \rho_{\mathrm{s}} / M_{\mathrm{s}}
$$

Combining eqs 8 and 9 gives the relation between swelling factor and molar solvent concentration

$$
F=\frac{\rho_{\mathrm{s}}}{\rho_{\mathrm{s}}-c_{\mathrm{s}} M_{\mathrm{s}}}
$$

A method-of-lines numerical method was applied to solve the convective diffusion problem, as illustrated in Figure 5. We have followed a similar procedure as Bisschops et al. ${ }^{69}$ where diffusion of solvent caused by a gradient in solvent concentration is described on a moving grid. The initial thickness of the pure polymer film at $t=0$ is $\delta(0)$. As solvent is penetrating from the surface into the polymer, the polymer starts to swell, causing the polymer/solvent interface to move in opposite direction. The initial polymer slab is divided in a number of layers of equal thickness, $\Delta x_{0}$, at $t=0$; hence, the grid points are at equal distance. During swelling, at $0<t<$ $t_{\text {end }}$ these grid points move with the same velocity as the polymer moves outward (the moving grid) until solvent saturation at $t=t_{\text {end }}$. During swelling, the total film thickness, $\delta$, increases at a velocity equal to that of the interface. At $t=0$, diffusion of solvent starts to occur under the influence of a concentration gradient, with an equilibrium concentration $c_{s}^{*}$ at interface, and $c_{s}=0$ everywhere else, as depicted in Figure 5, left pane. This equilibrium concentration is calculated from the final swelling factor using eq 10 . At $0<t<t_{\text {end }}$ a solvent concentration gradient exists as schematically shown in the center pane of Figure 5. Note that eq 10 implies that a higher solvent concentration corresponds to a higher swelling coefficient. Therefore, the different polymer layers are expanding at different rates as long as a concentration gradient is present.

The convective diffusion equation to be solved differs from the usual form, as it has to account for simultaneous swelling. Consider a volume element at time $t$ and solvent concentration $c$ (we drop the subscript $\mathrm{s}$ for the remainder of this derivation) of volume $V=a \Delta x$, where $\Delta x$ is its thickness $\left(\Delta x_{0}\right.$ at $\left.t=0\right)$ and $a$ is its cross-sectional area. This volume element contains $c V$ moles of solvent. At time $t+\mathrm{d} t$, the solvent concentration is $c+\Delta c$, the thickness is $x+\Delta x^{\prime}$, and the amount of solvent is $c V+\Delta(c V)$. By evaluating this change in amount of solvent to the difference in influx and outflux at both sides of the volume element, during $\Delta t$ through surface $a$, we can write the finite difference equation

$$
\Delta(c V)=a\left(\left(-D \frac{\mathrm{d} c}{\mathrm{~d} x}\right)_{x+\Delta x}-\left(-D \frac{\mathrm{d} c}{\mathrm{~d} x}\right)_{x}\right) \Delta t
$$

With $\Delta t \rightarrow 0$ and substituting $V$, we obtain the first order differential equation

$$
\frac{\mathrm{d}(c \Delta x)}{\mathrm{d} t}=\left(-D \frac{\mathrm{d} c}{\mathrm{~d} x}\right)_{x+\Delta x}-\left(-D \frac{\mathrm{d} c}{\mathrm{~d} x}\right)_{x}
$$

In the absence of swelling, $\Delta x$ remains constant during the diffusion process and equal to $\Delta x_{0}$. With an infinite number of lines, (i.e., letting $\Delta x_{0} \rightarrow 0$ ), eq 12 reduces to the usual secondorder differential equation for convective diffusion of eq 2 .

In the presence of swelling, we can make use of the relation between the swelling factor and thickness

$$
\Delta x=F \Delta x_{0}
$$

The relation between swelling factor and solvent concentration in eq 10 allows writing $c \Delta x$ as a function of $c$ only. After differentiation, the left-hand side of eq 12 then becomes

$$
\Delta x_{0}\left(1+\frac{M \rho c}{F(\rho-M c)^{2}}\right)^{-1} \frac{\mathrm{d} c}{\mathrm{~d} t}
$$

Thus, letting $\Delta x_{0} \rightarrow 0$, eq 12 can be written as the secondorder partial differential equation

$$
\frac{\mathrm{d} c(t, x)}{\mathrm{d} t}=\left\{1+\frac{M \rho c(t, x)}{F(\rho-M c(t, x))^{2}}\right\}^{-1} \frac{\mathrm{d}}{\mathrm{d} x}\left(D \frac{\mathrm{d} c(t, x)}{\mathrm{d} x}\right)
$$

Note that both the diffusion coefficient $D$ (eq 7) and the swelling factor $F$ (eq 10) are functions of $t$ and $x$. This formulation of the convective diffusion equation accounts for two effects of swelling: (1) the stretching of the polymer layers that reduces the solvent concentration gradient; (2) the dilution of penetrant in the polymer by an increase in volume. The new diffusion-swelling model is more general than eq 3 and includes both non-Fickian and Fickian diffusion. For Fickian diffusion, one would assume constant $D$ and film thickness, which yields results identical to eq 2 .

The partial differential equation in eq 14 was solved using the method-of-lines as illustrated in Figure 5 with the following boundary conditions

$$
\begin{array}{ll}
t=0,0<x<\delta: & c=0 \\
t \geq 0, x=\delta(t): & c=c^{*} \\
t \geq 0, x=0: & \frac{\mathrm{d} c}{\mathrm{~d} x}=0
\end{array}
$$

It is important to note that similar to what was assumed by Bisschops et al., ${ }^{69}$ the concentration gradient is defined with respect to a moving grid. Thus, the finite difference version of eq 14 is solved for each layer of increasing thickness, and at each integration step the thickness of the slabs is updated.

Finally, we derive a relation between the concentration of solvent and the measured IR absorbance, as an alternative to eq 3, which assumes a constant diffusion coefficient and no sample swelling. The starting point for this derivation is eq 15 , relating the absorbance of the IR band of the penetrating solvent over time, $A(t)$, to the changing concentration profile in the film, $c(t, x)$. This relation is based on the decay of the evanescent electric field strength associated with the IR beam ${ }^{21}$

$$
A(t)=K \int_{0}^{\delta} c(t, x) \mathrm{e}^{-2 \gamma x} \mathrm{~d} x
$$

Using an expression for the relative IR absorbance and concentration allows us to use a simplified representation (eq 15 ) instead of the full equation used by Fieldson and Barbari. ${ }^{21}$ 

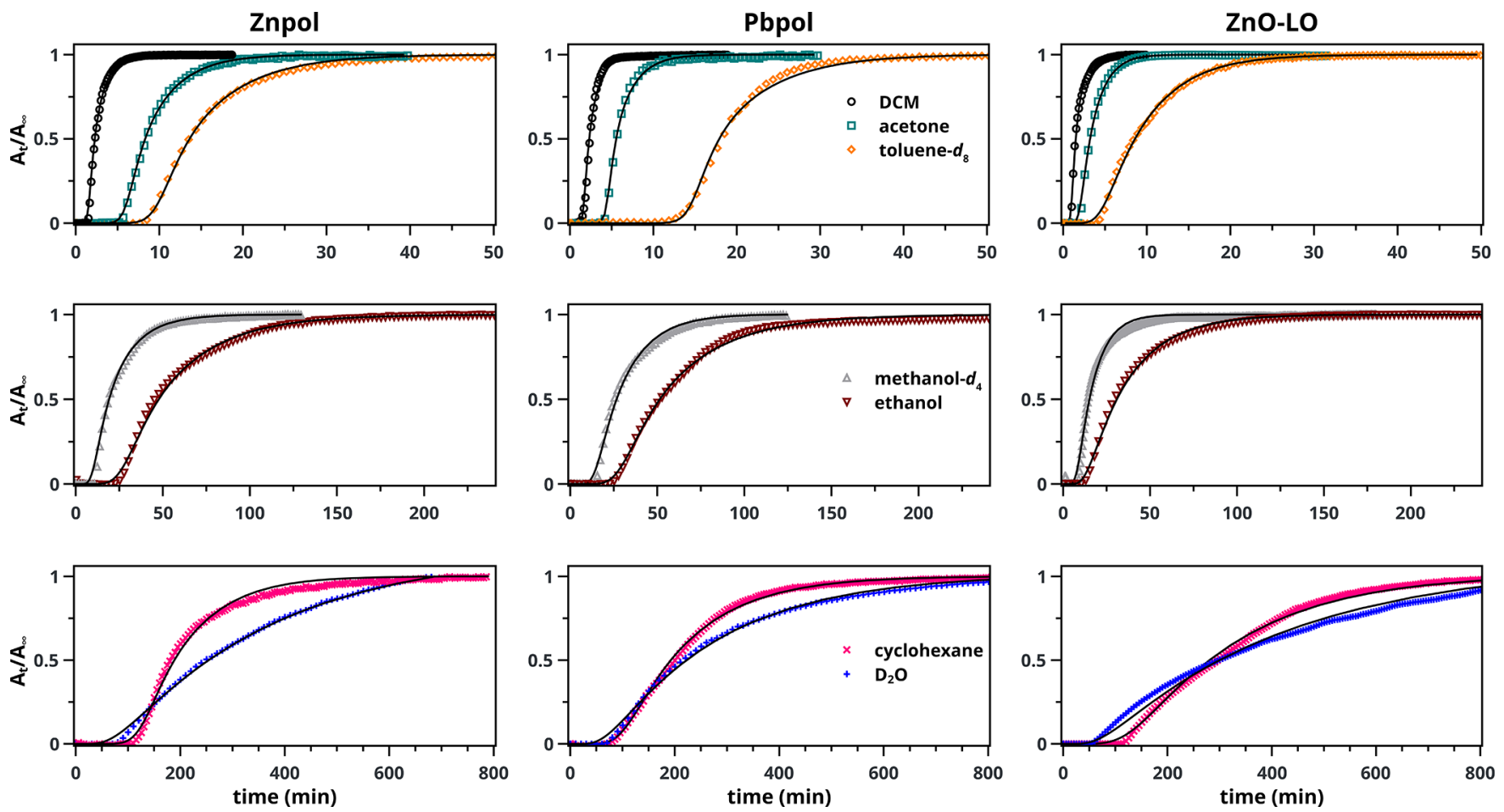

Figure 6. Diffusion profiles of seven solvents in Pbpol, Znpol, and ZnO-LO films of 135-160 $\mu$ m thickness and a metal ion concentration in the polymer films of roughly $420 \mathrm{mM}$. Solid black lines represent best fits of the two-parameter diffusion-swelling model (eq 7) as employed in the convective diffusion equation (eq 14).

In eq 15 , the factor $K$ is determined by several optical properties like the extinction coefficient and refractive indices. Using the ratio $A(t) / A_{\infty}$, factor $K$ vanishes, while the parameter $\gamma$ is still defined as in eq 4 . Because of the fast exponential decay of the evanescent wave $\left(\mathrm{e}^{-2 \gamma x}\right)$, the concentration at the bottom of the film $c(t, x=0)$ accounts for the measured absorbance $A(t)$. The relative change of solvent concentration over time can now be expressed as

$$
\frac{c(t, 0)}{c(\infty, 0)}=\frac{A(t)}{A(\infty)}
$$

In this model, $A(t)$ is the area of an IR band of the penetrant at time $t$, and $A(\infty)$ is the band area when the sample is completely saturated with penetrant.

Application of the Diffusion-Swelling Model. Figure 6 shows the experimental solvent profiles and fitted curves according to the diffusion-swelling model with nonconstant diffusion coefficients. In all cases, an excellent agreement was found between the experimental and fitted curves. The values obtained for $D_{\text {eq }}$ are listed in Table 2. The trend in the values of solvent diffusion coefficients is similar to those determined by measurements of paint swelling on polymerized linseed oil films reported by Stolow. ${ }^{70}$ For water, the measured diffusion coefficients are of the same order of magnitude as the values reported for water diffusion in pigmented alkyd paints ${ }^{37}$ and vapor diffusion in historical samples of Prussian blue and basic lead carbonate paints ${ }^{33}$ (in the range $(1.2-7.7) \times 10^{-13} \mathrm{~m}^{2} / \mathrm{s}$ ).

An important achievement of the diffusion-swelling model is that unlike the (adapted) Fickian model, it gives an adequate prediction of $\tau_{\mathrm{d}}$. The values for $\alpha$ obtained are listed in Table S4 and range between 0 and -19 . The fact that $\alpha \leq 0$ indicates that the diffusion rate increases with decreasing polymer fraction $\phi$ during the transition from dry to swollen polymer.
The relation between $D$ and $\phi$ for solvent diffusion in $\mathrm{Znpol}$ is shown in Figure 7 (for Pbpol and $\mathrm{ZnO}-\mathrm{LO}$, see Figure S5),

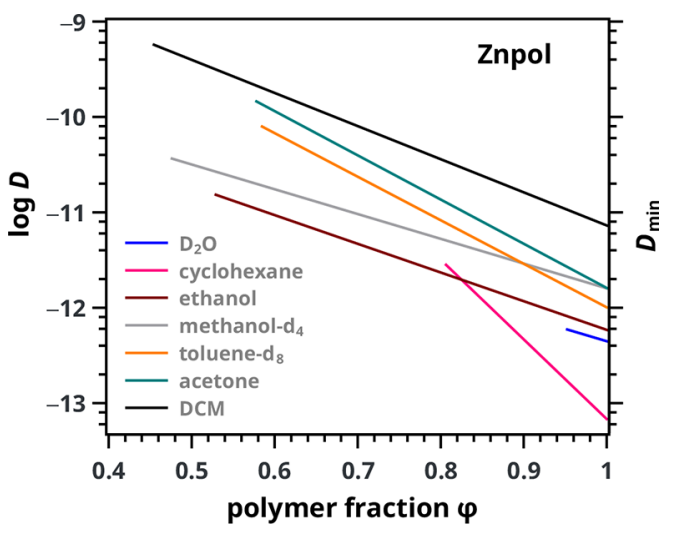

Figure 7. Diffusion coefficient in Znpol film versus polymer fraction $\phi$, calculated using the diffusion-swelling model, eq 8, using parameters in Tables S3 and S4.

which also highlights the significant differences in the equilibrium swelling factor, with $f_{\text {eq }}$ ranging between 0.05 for $\mathrm{D}_{2} \mathrm{O}$ and 1.2 for DCM. The minimal values for $D\left(D_{\min }\right)$, in the dry state, are in the order of $10^{-13} \mathrm{~m}^{2} \mathrm{~s}^{-1}$, which is still higher than typical diffusion coefficients found for glassy polymers. ${ }^{71}$ This finding is in agreement with the $T_{\mathrm{g}}$ values close to room temperature that we measured for dry Znpol, $\mathrm{Pbpol}$, and $\mathrm{ZnO}-\mathrm{LO}$. In general, similar diffusion coefficients (varying less than a factor of 2) were found for all three investigated model paint systems, indicating that small differences in chemical composition do not significantly affect the diffusivity. It is especially noteworthy that the introduction of $50 \mathrm{wt} \%$ of pigment in $\mathrm{ZnO}-\mathrm{LO}$ does not lead to strongly 
altered diffusion behavior. This result indicates that in intact oil paint films (i.e., paint films without cracks) solvent diffusion around pigment particles is relatively fast, and the rate of diffusion is mostly determined by the properties of the organic polymer matrix.

Figure S6 compares the diffusion coefficients obtained using the adapted ideal model $D_{\text {Fick }}$ and those at equilibrium swelling obtained using the diffusion-swelling model $\left(D_{\text {eq }}\right)$. In the adapted ideal model (eq 5), swelling effects above or inside the measurement volume after the first detection of solvent signal that slow down solvent migration are not taken into account. The diffusion-swelling model does incorporate this swelling, and therefore one expects $D_{\text {eq }}>D_{\text {Fick. }}$. Indeed, $D_{\text {eq }}$ is consistenly larger than $D_{\text {Fick }}$, with the difference being largest for the most swelling solvent (DCM) and very small for the least swelling solvent $\left(\mathrm{D}_{2} \mathrm{O}\right)$. These observations show that even though only minor swelling occurs after solvent signal is first detected, a variable diffusion coefficient is necessary to accurately model the solvent diffusion process close to equilibrium swelling. However, the simple adapted ideal model can still be used to calculate a reasonable approximation of the solvent diffusion coefficient. Tables S3 and S4 list the obtained diffusion parameters $D_{\text {eq }}$ and $\alpha$ from eq 7 values with their $95 \%$ confidence intervals for all paint models.

Having established that the swelling-diffusion model accurately describes solvent transport in oil paint systems, we can now compare diffusion parameters of the different solvents. The complexity of the solvent diffusion process is illustrated by the fact that some of the faster diffusing solvents like DCM and toluene have the highest molecular weight and that toluene and cyclohexane have very different diffusion rates despite having a similar structure. Moreover, the two slowest diffusing solvents-water and cyclohexane-are the most and least polar solvents and have the smallest and largest molecular radius in the set.

According to Amsden, ${ }^{64}$ the parameter $\alpha$ of eq 6 , which determines how strongly the solvent diffusion coefficient changes with polymer fraction, is related to the molecular radius of the diffusing molecule. The fitted values of $\alpha$ are plotted versus the molecular radius of the solvent molecules in Figure S7. With this solvent set, we did not find a significant correlation between molecular radius and $\alpha$. Because our set of solvent consists of a limited range of molecular radii and very diverse solvent properties, it is necessary to consider different solvent properties to explain the differences in diffusion behavior. Stolow ${ }^{70}$ correlated kinematic viscosity $\nu$ (absolute viscosity $\eta$ divided by density $\rho$ ) and the diffusion coefficient. As illustrated in Figure S8, our findings confirm this correlation, with the more slowly flowing solvents having the lowest diffusion coefficients. Water deviates from this trend with a diffusion rate that is much slower than what is expected based on its kinematic viscosity.

Historically, equilibrium swelling data have been used to quantify diffusion behavior in paints. ${ }^{70}$ Additionally, a high degree of swelling is associated with an increased risk of pigment loss during oil paint cleaning treatments. ${ }^{30}$ Figure 8 shows a logarithmic plot of $D_{\text {eq }}$ versus $f_{\text {eq }}$, showing that solvents with a high swelling capacity are indeed generally diffusing faster. Alcohols exhibit relatively strong swelling compared to their moderately fast diffusion. These results confirm that swelling studies can be a useful indication of diffusion behavior of solvents in linseed oil-based paints.

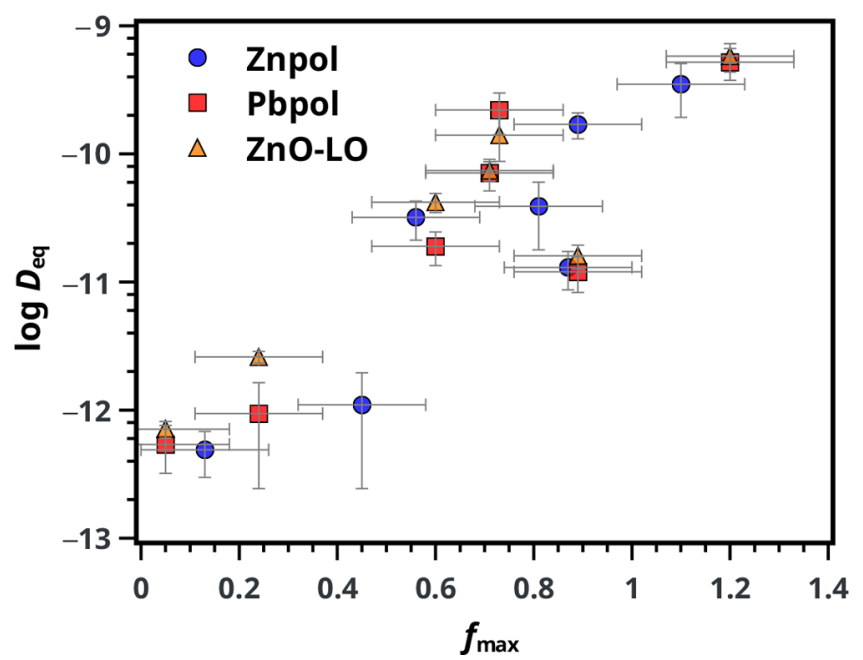

Figure 8. Correlation of equilibrium swelling factor, $f_{\text {eq }}$, with $D_{\text {eq }}$. Lower swelling generally leads to slower diffusion. Error bars calculated as described in the Supporting Information.

However, it is important to emphasize that accurately describing diffusion phenomena in paint is not straightforward.

The experiments described in this work have practical implications for paintings restoration. Disregarding potential reactivity between the solvent and paint components, it is often desired to minimize mechanical stress and limit the volume of paint material that is affected by a solvent during cleaning. In this light, both water and aliphatic hydrocarbons like cyclohexane offer a combination of two useful properties: low swelling power and slow diffusion. As water and aliphatic hydrocarbons are at opposite ends of the polarity scale, it should be possible to remove a broad range of soiling materials from the surface of oil paintings with these two solvents. The risk of mechanical damage can be further reduced by making use of tailored gel systems for oil paint cleaning. ${ }^{72,73}$ To this end, we would encourage the development of gel systems that can be loaded with aliphatic solvents, solvent mixtures, or even microemulsions that allow precise tuning of solvent polarity. Finally, the methods described here can be extended to study paint swelling and solvent diffusion for porous paint and mixtures of solvents.

\section{CONCLUSION}

Paintings are exposed to organic solvents and aqueous solutions during restoration treatments, which may lead to chemical alterations within the paint layers and altered viscoelastic properties of the paint. We have characterized linseed oil based binding medium models with DMA and found viscoelastic properties similar to classical ionomers. Ionomers containing zinc show gradual broadening of $\tan \delta$ with increasing metal content whereas lead ionomers show characteristic behavior of matrix- and ion-cluster relaxation. In all cases, $T_{\mathrm{g}}$ is only weakly influenced by metal content.

Accurate concentration profiles of solvents and water in model paint samples were measured successfully using ATRFTIR spectroscopy. Both an adapted Fickian diffusion model and a diffusion-swelling model incorporating the effect of film swelling on diffusion were used to describe the experimental data. In contrast to the adapted Fickian diffusion model, the considerable delay time observed for the relatively thick films was successfully predicted by the diffusion-swelling model. 
With the diffusion-swelling model, it was found that the solvent diffusion coefficients of solvents increase during paint swelling. No single solvent parameter was sufficient to explain the measured trend in solvent swelling or diffusion rate. However, strongly swelling solvents (e.g., acetone) generally diffuse faster than weakly swelling solvents (e.g., water).

\section{ASSOCIATED CONTENT}

\section{S Supporting Information}

The Supporting Information is available free of charge on the ACS Publications website at DOI: 10.1021/acs.macromol.8b00890.

Details on the composition of samples, the analysis of DMA and FTIR spectroscopy data and the procedure for error estimation; additional data tables with fit parameters and figures showing the correlation between fit parameters and solvent/polymer properties (PDF)

\section{AUTHOR INFORMATION}

\section{Corresponding Authors}

*E-mail: c.l.m.baij@uva.nl (L.B.).

*E-mail: j.j.hermans@uva.nl (J.J.H.).

\section{ORCID}

Lambert Baij: 0000-0002-9196-2955

Joen J. Hermans: 0000-0002-9446-9904

\section{Author Contributions}

L.B. and J.J.H. contributed equally.

\section{Notes}

The authors declare no competing financial interest.

\section{ACKNOWLEDGMENTS}

The authors thank Dr. Hans Poulis, Lijing Xue, and Frans van Oostrum for use of the DMA equipment and Prof. Kim McAuley for useful discussions. This research was carried out within the framework of the NANORESTART project funded by the European Union's Horizon 2020 research and innovation program under Agreement No. 646063.

\section{REFERENCES}

(1) Baij, L.; Hermans, J.; Keune, K.; Iedema, P. Time-dependent ATR-FTIR studies on fatty acid diffusion and the formation of metal soaps in oil paint model systems. Angew. Chem., Int. Ed. 2018, 57 (25), 7351-7354.

(2) Khandekar, N. A survey of the conservation literature relating to the development of aqueous gel cleaning on painted and varnished surfaces. Stud. Conserv. 2000, 45, 10-20.

(3) Phenix, A.; Sutherland, K. The cleaning of paintings: effects of organic solvents on oil paint films. Stud. Conserv. 2001, 46, 47-60.

(4) Mecklenburg, M. F.; Charola, A. E.; Koestler, R. J. New Insights into the Cleaning of Paintings: Proceedings from the Cleaning 2010 International Conference, Universidad Politécnica de Valencia and Museum Conservation Institute. Smithsonian Contributions to Museum Conservation 2013, 3, 1-243.

(5) Baij, L.; Keune, K.; Hermans, J. J.; Noble, P.; Iedema, P. D. In Gels in the Conservation of Art; Angelova, L. V., Ormsby, B., Townsend, J. H., Wolbers, R., Eds.; Archetype Publications: London, 2017; pp 316-321.

(6) Porter, N. A.; Caldwell, S. E.; Mills, K. A. Mechanisms of free radical oxidation of unsaturated lipids. Lipids 1995, 30, 277-290.

(7) Porter, N. A.; Weber, B. A.; Weenen, H.; Khan, J. A. Autoxidation of polyunsaturated lipids. Factors controlling the stereochemistry of product hydroperoxides. J. Am. Chem. Soc. 1980, 102, 5597-5601.
(8) Yin, H.; Porter, N. A. New insights regarding the autoxidation of polyunsaturated fatty acids. Antioxid. Redox Signaling 2005, 7, 170184.

(9) Yin, H.; Xu, L.; Porter, N. A. Free radical lipid peroxidation: mechanisms and analysis. Chem. Rev. 2011, 111, 5944-5972.

(10) Gardner, H. W. Oxygen radical chemistry of polyunsaturated fatty acids. Free Radical Biol. Med. 1989, 7, 65-86.

(11) Soucek, M. D.; Khattab, T.; Wu, J. Review of autoxidation and driers. Prog. Org. Coat. 2012, 73, 435-454.

(12) Lazzari, M.; Chiantore, O. Drying and oxidative degradation of linseed oil. Polym. Degrad. Stab. 1999, 65, 303-313.

(13) Mallégol, J.; Lemaire, J.; Gardette, J. L. Drier influence on the curing of linseed oil. Prog. Org. Coat. 2000, 39, 107-113.

(14) Van Gorkum, R.; Bouwman, E. The oxidative drying of alkyd paint catalysed by metal complexes. Coord. Chem. Rev. 2005, 249, $1709-1728$

(15) Hubert, J. C.; Venderbosch, R. A. M.; Muizebelt, W. J.; Klaasen, R. P.; Zabel, K. H. Mechanistic study of drying of alkyd resins using $(\mathrm{Z}, \mathrm{Z})$-and (E, E)-3, 6-nonadiene as model substances. Prog. Org. Coat. 1997, 31, 331-340.

(16) Oyman, Z. O.; Ming, W.; Micciche, F.; Oostveen, E.; Van Haveren, J.; van Der Linde, R. A promising environmentally-friendly manganese-based catalyst for alkyd emulsion coatings. Polymer 2004, 45, 7431-7436.

(17) Oakley, L.; Casadio, F.; Shull, K.; Broadbelt, L. Microkinetic modeling of the autoxidative curing of an alkyd and oil-based paint model system. Appl. Phys. A: Mater. Sci. Process. 2015, 121, 869.

(18) Hermans, J. J.; Keune, K.; van Loon, A.; Iedema, P. D. An infrared spectroscopic study of the nature of zinc carboxylates in oil paintings. J. Anal. At. Spectrom. 2015, 30, 1600-1608.

(19) Hermans, J. J.; Keune, K.; van Loon, A.; Corkery, R. W.; Iedema, P. D. Ionomer-like structure in mature oil paint binding media. RSC Adv. 2016, 6, 93363-93369.

(20) Van Der Wel, G. K.; Adan, O. C. G. Moisture in organic coatings - a review. Prog. Org. Coat. 1999, 37, 1-14.

(21) Fieldson, G. T.; Barbari, T. A. The use of FTi.r.-a.t.r. spectroscopy to characterize penetrant diffusion in polymers. Polymer 1993, 34, 1146-1153.

(22) Hong, S. U.; Barbari, T. A.; Sloan, J. M. Multicomponent diffusion of methyl ethyl ketone and toluene in polyisobutylene from vapor sorption FTIR-ATR spectroscopy. J. Polym. Sci., Part B: Polym. Phys. 1998, 36, 337-344.

(23) Sammon, C.; Yarwood, J.; Everall, N. A FTIR-ATR study of liquid diffusion processes in PET films: Comparison of water with simple alcohols. Polymer 2000, 41, 2521-2534.

(24) Guo, J.; Barbari, T. A. Unified dual mode description of small molecule sorption and desorption kinetics in a glassy polymer. Macromolecules 2009, 42, 5700-5708.

(25) Farinas, K. C.; Doh, L.; Venkatraman, S.; Potts, R. O. Characterization of solute diffusion in a polymer using ATR-FTIR spectroscopy and bulk transport techniques. Macromolecules 1994, 27, $5220-5222$.

(26) Dias, M.; Hadgraft, J.; Raghavan, S. L.; Tetteh, J. The effect of solvent on permeant diffusion through membranes studied using ATR-FTIR and chemometric data analysis. J. Pharm. Sci. 2004, 93, 186-196.

(27) Van Alsten, J. G. Ionic and Chain Interdiffusion and Interfacial Strength Development in Ionomers of Poly(ethylene- co -methacrylic acid). Macromolecules 1996, 29, 2163-2168.

(28) Fleming, O. S.; Chan, K. L. A.; Kazarian, S. G. High-pressure $\mathrm{CO}_{2}$-enhanced polymer interdiffusion and dissolution studied with in situ ATR-FTIR spectroscopic imaging. Polymer 2006, 47, 4649-4658.

(29) Stolow, N. Action of Solvents on Dried Linseed Oil Films. Nature 1957, 179, 579-580.

(30) Phenix, A. The Swelling of Artists' Paints in Organic Solvents. Part 1, a Simple Method for Measuring the In-Plane Swelling of Unsupported Paint Films. J. Am. Inst. Conserv. 2002, 41, 43. 
(31) Ward, S. A.; Pethrick, R. A. Positron annihilation lifetime spectroscopy and diffusion studies of large molecule penetrants into alkyd resins. Prog. Org. Coat. 2012, 75, 509-526.

(32) Sharma, V.; Banait, J. S.; Kundu, P. P. Swelling kinetics of linseed oil-based polymers. J. Appl. Polym. Sci. 2009, 111, 1816-1827.

(33) Chan, T. Y. A.; Odlyha, M. The effect of relative humidity and pigment type on paint films. Thermochim. Acta 1995, 269-270, 755767.

(34) Nguyen, T.; Bentz, D.; Byrd, E. Method for measuring water diffusion in a coating applied to a substrate. J. Coat. Technol. 1995, 67, $37-46$.

(35) Liu, B.; Li, Y.; Lin, H.; Cao, C.-n. Effect of PVC on the diffusion behaviour of water through alkyd coatings. Corros. Sci. 2002, 44, 2657-2664.

(36) Xu, Y.; Yan, C.; Ding, J.; Gao, Y.; Cao, C. Water vapour in the coatings of alkyd and polyurethane varnish. Prog. Org. Coat. 2002, 45, 331-339.

(37) van der Zanden, A.; Goossens, E. The measurement of the diffusion coefficient and the sorption isotherm of water in paint films. Chem. Eng. Sci. 2003, 58, 1521-1530.

(38) Saarinen, V.; Kreuer, K. D.; Schuster, M.; Merkle, R.; Maier, J. On the swelling properties of proton conducting membranes for direct methanol fuel cells. Solid State Ionics 2007, 178, 533-537.

(39) Hallinan, D. T.; De Angelis, M. G.; Giacinti Baschetti, M.; Sarti, G. C.; Elabd, Y. A. Non-Fickian Diffusion of Water in Nafion. Macromolecules 2010, 43, 4667-4678.

(40) Hickner, M. A. Water-mediated transport in ion-containing polymers. J. Polym. Sci., Part B: Polym. Phys. 2012, 50, 9-20.

(41) Dishari, S. K.; Hickner, M. A. Antiplasticization and water uptake of nafion thin films. ACS Macro Lett. 2012, 1, 291-295.

(42) Davis, E. M.; Stafford, C. M.; Page, K. A. Elucidating water transport mechanisms in Nafion thin films. ACS Macro Lett. 2014, 3, $1029-1035$.

(43) Yao, K. Z.; Shaw, B. M.; Kou, B.; McAuley, K. B.; Bacon, D. W. Modeling Ethylene/Butene Copolymerization with Multi-site Catalysts: Parameter Estimability and Experimental Design. Polym. React. Eng. 2003, 11, 563-588.

(44) Podgorski, L.; Merlin, A.; Saiter, J. Natural and artificial ageing of an alkyd based wood finish: Calorimetric investigations. J. Therm. Anal. 1994, 41, 1319-1324.

(45) Hedley, G.; Odlyha, M.; Burnstock, A.; Tillinghast, J.; Husband, C. A study of the mechanical and surface properties of oil paint films treated with organic solvents and water. J. Therm. Anal. 1991, 37, 2067-2088

(46) Phenix, A.; Mechanical, T.; Paints, O.; Phenix, A. Thermal Mechanical Transitions in Artists' Oil Paints and Selected Conservation Materials: a Study by Dynamic Mechanical Analysis (DMA). AIC Paintings Specialty Group Postprints 2009, 22, 72-89.

(47) Sanches, D.; Ramos, A. M.; Coelho, J. F. J.; Costa, C. S. M. F.; Vilarigues, M.; Melo, M. J. Correlating thermophysical properties with the molecular composition of 19th century chrome yellow oil paints. Polym. Degrad. Stab. 2017, 138, 201-211.

(48) Ploeger, R.; Scalarone, D.; Chiantore, O. Thermal analytical study of the oxidative stability of artists' alkyd paints. Polym. Degrad. Stab. 2009, 94, 2036-2041.

(49) Mosiewicki, M. A.; Rojas, O.; Sibaja, M. R.; Borrajo, J.; Aranguren, M. I. Aging study of linseed oil resin/styrene thermosets and their composites with wood flour. Polym. Int. 2007, 56, 875-881.

(50) Boquillon, N.; Fringant, C. Polymer networks derived from curing of epoxidisedlinseed oil: influence of different catalysts and anhydride hardeners. Polymer 2000, 41, 8603-8613.

(51) Earnest, T. R.; MacKnight, W. J. Infrared Studies of Hydrogen Bonding in Ethylene-Methacrylic Acid Copolymers and Ionomers. Macromolecules 1980, 13, 844-849.

(52) Eisenberg, A. Clustering of Ions in Organic Polymers. A Theoretical Approach. Macromolecules 1970, 3, 147-154.

(53) Eisenberg, A.; Hird, B.; Moore, R. B. A new multiplet-cluster model for the morphology of random ionomers. Macromolecules 1990, 23, 4098-4107.
(54) Hermans, J. J.; Keune, K.; van Loon, A.; Iedema, P. D.; van Loon, A.; Iedema, P. D. The crystallization of metal soaps and fatty acids in oil paint model systems. Phys. Chem. Chem. Phys. 2016, 18, 10896-10905.

(55) Park, J.; Eslick, J.; Ye, Q.; Misra, A.; Spencer, P. The influence of chemical structure on the properties in methacrylate-based dentin adhesives. Dent. Mater. 2011, 27, 1086-1093.

(56) Hill, L. W. Calculation of crosslink density in short chain networks. Prog. Org. Coat. 1997, 31, 235-243.

(57) Weiss, R. A.; Fitzgerald, J. J.; Kim, D. Viscoelastic behavior of plasticized sulfonated polystyrene ionomers. Macromolecules 1991, 24, $1064-1070$

(58) Giacinti Baschetti, M.; Piccinini, E.; Barbari, T. A.; Sarti, G. C. Quantitative Analysis of Polymer Dilation during Sorption Using FTIR-ATR Spectroscopy. Macromolecules 2003, 36, 9574-9584.

(59) Browne, F. Swelling of paint-films in water VII: Swelling of Linseed Oil Paints in Water and Organic Liquids. Forest Products Journal 1956, 5, 312-318.

(60) Zumbühl, S. Parametrization of the solvent action on modern artists' paint systems. Stud. Conserv. 2014, 59, 24-37.

(61) Phenix, A. Effects of organic solvents on artists' oil paint films: swelling. Smithsonian Contributions to Museum Conservation 2013, 3, 69-76.

(62) Brannon-Peppas, L.; Peppas, N. A. Equilibrium swelling behavior of pH-sensitive hydrogels. Chem. Eng. Sci. 1991, 46, 715722.

(63) Edwards, D. A. Non-Fickian Diffusion in Thin Polymer Films. J. Polym. Sci., Part B: Polym. Phys. 1996, 34, 981-997.

(64) Amsden, B. Solute Diffusion within Hydrogels. Mechanisms and Models. Macromolecules 1998, 31, 8382-8395.

(65) Masaro, L.; Zhu, X. X. Physical models of diffusion for polymer solutions, gels and solids. Progress in Polymer Science; Elsevier: 1999; Vol. 24, pp 731-775.

(66) De Kee, D.; Liu, Q.; Hinestroza, J. Viscoelastic (Non-Fickian) Diffusion. Can. J. Chem. Eng. 2005, 83, 913-929.

(67) Vrentas, J. S.; Duda, J. L. Diffusion in polymer-solvent systems. I. Reexamination of the free-volume theory. J. Polym. Sci., Polym. Phys. Ed. 1977, 15, 403-416.

(68) Vrentas, J. S.; Duda, J. L. Diffusion in polymer-solvent systems. II. A predictive theory for the dependence of diffusion coefficients on temperature, concentration, and molecular weight. J. Polym. Sci., Polym. Phys. Ed. 1977, 15, 417-439.

(69) Bisschops, M. a. T.; Luyben, K. C. a. M.; van der Wielen, L. A. M. Generalized Maxwell-Stefan Approach for Swelling Kinetics of Dextran Gels. Ind. Eng. Chem. Res. 1998, 37, 3312-3322.

(70) Stolow, N. The action of solvents on drying-oil films: parts I and II. J. Oil Colour Chemists' Assoc. 1957, 40, 377-402.

(71) Guo, J.; Barbari, T. A. A Dual Mode, Local Equilibrium Relaxation Model for Small Molecule Diffusion in a Glassy Polymer. Macromolecules 2008, 41, 238-245.

(72) Duncan, T. T.; Berrie, B. H.; Weiss, R. G. Soft, Peelable Organogels from Partially Hydrolyzed Poly(vinyl acetate) and Benzene-1,4-diboronic Acid: Applications to Clean Works of Art. ACS Appl. Mater. Interfaces 2017, 9, 28069-28078.

(73) Baglioni, M.; Domingues, J. A. L.; Carretti, E.; Fratini, E.; Chelazzi, D.; Giorgi, R.; Baglioni, P. Complex Fluids Confined into Semi-interpenetrated Chemical Hydrogels for the Cleaning of Classic Art: A Rheological and SAXS Study. ACS Appl. Mater. Interfaces 2018, 10, 19162-19172. 